\title{
Psoralidin prevents caffeine-induced damage and abnormal differentiation of bone marrow mesenchymal stem cells via the classical estrogen receptor pathway
}

\author{
Rong Hua ${ }^{1 \#}$, Jilong Zou ${ }^{2 \#}$, Ying $\mathrm{Ma}^{1}$, Xiaomei Wang ${ }^{1}$, Yao Chen ${ }^{1}$, Yuan $\mathrm{Li}^{3,4}$, Jianyang $\mathrm{Du}^{5} \wedge$ \\ ${ }^{1}$ Department of Clinical Pharmacy, Department of Pharmacy, Taizhou People's Hospital, the Hospital Affiliated 5 to Nantong University, Taizhou, \\ China; ${ }^{2}$ Department of Orthopaedics, the First Affiliated Hospital of Harbin Medical University, Harbin, China; ${ }^{3}$ Department of Pharmacology, School \\ of Pharmaceutical Sciences, Cheeloo College of Medicine, Shandong University, Jinan, China; ${ }^{4}$ Suzhou Research Institute, Shandong University, \\ Suzhou, China; ${ }^{5}$ Department of Neurosurgery, Shandong Provincial Hospital Affiliated to Shandong First Medical University, Jinan, China \\ Contributions: (I) Conception and design: J Du, Y Li; (II) Administrative support: J Du, Y Li; (III) Provision of study materials or patients: R Hua, \\ J Zou; (IV) Collection and assembly of data: R Hua, J Zou, Y Ma, X Wang, Y Chen; (V) Data analysis and interpretation: R Hua, J Zou, Y Ma, X \\ Wang, Y Chen; (VI) Manuscript writing: All authors; (VII) Final approval of manuscript: All authors. \\ \#These authors contributed equally to this work. \\ Correspondence to: Jianyang Du. Department of Neurosurgery, Shandong Provincial Hospital Affiliated to Shandong First Medical University, Jinan \\ 250021, China. Email: jianyangdu@126.com; Yuan Li. Department of Pharmacology, School of Pharmaceutical Sciences, Cheeloo College of \\ Medicine, Shandong University, Jinan 250012, China. Email: yuanli@sdu.edu.cn.
}

Background: Caffeine is broadly present in tea, coffee, and cocoa, and is commonly consumed. The bone microenvironment might be damaged by excessive caffeine, which has been shown to exert negative effects on human health. In this study, we sought to determine whether excessive caffeine could damage the biological functions of bone marrow mesenchymal stem cells (BMSCs) and induce bone loss in mice, and further investigate effective therapeutic methods.

Methods: BMSCs were treated with different concentrations of caffeine $(0.01,0.05,0.1,0.5$, and $1.0 \mathrm{mM}$ ) for $48 \mathrm{~h}$. Cell counting kit-8 (CCK-8) assay, colony formation assay, wound healing assay, and quantitative real-time polymerase chain reaction (qRT-PCR) analysis were performed to detect the cell viability, proliferation, migration, and pluripotency of BMSCs, respectively. Alizarin red S (ARS) staining, alkaline phosphatase (ALP) staining, oil red O (ORO) staining, and qRT-PCR assay were applied to assess the osteogenic and adipogenic differentiation of BMSCs. BMSCs were treated with caffeine and further exposed to different concentrations of psoralidin (PL) $(0.01,0.1,1$, and $10 \mu \mathrm{M})$ for $48 \mathrm{~h}$. Microcomputed tomography $(\mu \mathrm{CT})$ scanning was used to evaluate the bone mass of mice. $7 \alpha-(7-((4,4,5,5,5-$ Pentafluoropentyl)-sulfiny)nonyl)estra-1,3,5(10)-triene-3,17 $\beta$-diol (ICI 182,780, ICI) was applied to examine whether the classical estrogen receptor (ER) pathway was involved.

Results: The CCK- 8 assay, colony formation assay, wound healing assay, and qRT-PCR analysis indicated that caffeine $(0.01,0.05,0.1,0.5,1.0 \mathrm{mM})$ attenuated the cell viability, proliferation, migration and pluripotency of BMSCs, respectively, in a concentration-dependent manner. Caffeine treatment inhibited osteogenic differentiation but promoted adipogenic differentiation of BMSCs in a dose-dependent manner. Furthermore, ARS staining, ALP staining, ORO staining, and qRT-PCR assay showed that excessive caffeine induced bone loss and osteoporosis (OP) in mice by regulating the osteogenesis and adipogenesis of BMSCs. Also, PL treatment could reverse the caffeine-induced dysfunctions and aberrant differentiation of BMSCs via the ER pathway.

^ ORCID: Yuan Li, 0000-0002-4552-5180; Jianyang Du, 0000-0001-9682-4183. 
Conclusions: Our results revealed a novel molecular mechanism for the therapeutic effects of PL in treating excessive caffeine-induced OP, which might shed new light on the clinical application of PL for caffeine-related OP.

Keywords: Psoralidin; caffeine; differentiation; bone marrow mesenchymal stem cells (BMSCs); estrogen receptor pathway

Submitted Jun 02, 2021. Accepted for publication Aug 05, 2021.

doi: 10.21037/atm-21-3153

View this article at: https://dx.doi.org/10.21037/atm-21-3153

\section{Introduction}

Osteoporosis (OP) is a systemic skeletal disease, which is characterized by microarchitectural deterioration of bone tissue, reduced bone mass, and a high susceptibility to bone fracture. The mortality of OP is increasing, and affects $34 \%$ of women worldwide (1). It has high rates of morbidity and mortality, and has become a global health problem (2). $\mathrm{OP}$ is a multi-factorial bone disease that is related to genetic factors, personal factors, gender, age, and lifestyle, including inadequate calcium intake, excessive alcohol, caffeine consumption, and smoking $(3,4)$. In the bone microenvironment, osteoblast-mediated bone formation and bone marrow fat play vital roles in maintaining bone homeostasis (5). OP is characterized by decreased bone formation and elevated fat accumulation. Maintenance of bone homeostasis requires dynamic bone remodeling balance, which is intricately controlled by certain microenvironments (6).

In recent decades, bone marrow mesenchymal stem cells (BMSCs) have emerged as potential microenvironmental modulators $(6,7)$. BMSCs, as a type of non-hematopoietic multipotent stem cell, can be differentiated into various cell types, including osteoblasts, adipocytes, and chondrocytes, under specific conditions $(8,9)$. An increasing number of studies have indicated that OP is mainly attributable to the imbalance between the osteogenesis and adipogenesis of BMSCs (10). According to these reports, the reduced osteoblast activity is mainly owing to the fact that BMSCs from patients with OP exhibit decreased capacity to differentiate into osteoblasts and increased capacity to differentiate into adipocytes (11). Thus, the shift between osteoblast and adipocyte differentiation of BMSCs plays a key role in the development of OP.

Caffeine (1,3,7-trimethylxanthine) is a type of central nervous system stimulant and the key xanthine alkaloid element in several beverages, particularly in coffee and tea (12). Caffeine is widely consumed in Europe and North America. The results of previous epidemiological studies have revealed that caffeine is a risk factor for OP, and results in reduced bone mineral density (BMD), a higher risk of bone fracture, and decreased calcium absorption in osteoporotic patients (13). However, little is known about the underlying mechanism of caffeine-related OP, and the effects of caffeine on the biological functions and differentiation of BMSCs have not been studied.

Psoralidin (PL), a prenylated coumestrol, is extracted from Psoralea corylifolia, and is usually applied in the treatment of OP $(14,15)$. Recent studies have indicated that PL could improve the osteogenic potentials of BMSCs (16). Also, in vivo studies have shown that PL could prevent bone loss in ovariectomy (OVX)-induced osteoporotic rats by inhibiting the formation of osteoclasts $(17,18)$. However, whether PL can regulate caffeineinduced dysfunction of BMSCs and caffeine-related OP remains unclear.

The effects of caffeine and PL in the biological functions of BMSCs, and their relationship, have not been clearly illustrated. In this study, we investigated the roles of caffeine in the biological functions and differentiation potentials of BMSCs in vitro and in vivo. We also demonstrated that PL played a protective role in the caffeine-induced dysfunction and aberrant differentiation of BMSCs via the classical estrogen receptor (ER) pathway. Consequently, our study suggested that PL could be used as a potential candidate in the treatment of caffeine-related OP by regulating the competitive relationship between the osteogenesis and adipogenesis of BMSCs. Our study provided a novel therapeutic method for the treatment of caffeine-caused bone loss.

We present the following article in accordance with the ARRIVE reporting checklist (available at https://dx.doi. org/10.21037/atm-21-3153). 


\section{Methods}

\section{BMSC isolation and culture}

BMSCs were harvested from 8-week-old female C57BL/6J mice (approximately $18-20 \mathrm{~g}$ ). The isolation of mouse BMSCs was performed according to the previous reports (19). The BMSCs were cultured in BMSC culture medium (Cyagen, USA) and maintained in a humidified incubator (Thermo, USA) of $5 \%$ carbon dioxide $\left(\mathrm{CO}_{2}\right)$ at $37^{\circ} \mathrm{C}$. The medium was replaced by fresh medium every 3 days. BMSCs reaching $60-70 \%$ confluence, were used for additional experiments.

\section{Drug treatment}

PL was purchased from Sigma-Aldrich (USA). PL $(10 \mathrm{mM})$ was dissolved in dimethyl sulfoxide (DMSO) and stored at $4{ }^{\circ} \mathrm{C}$ in a freezer (Haier, China), which were regarded as stock solutions. The stock solutions were then diluted with BMSC culture medium or differentiationinducing medium, which were regarded as working solutions in the further experiments. The concentration of DMSO in the working solutions was not higher than $0.2 \%$, which was known as a safe concentration. The cells were treated with different concentrations of PL (0.01, 0.1, 1.0, and $10 \mu \mathrm{M})$ for $48 \mathrm{~h}$. Caffeine was purchased from SigmaAldrich (USA), and the cells were treated with different concentrations of caffeine $(0.01,0.05,0.1,0.5$, and $1.0 \mathrm{mM})$ for $48 \mathrm{~h}$. The ER antagonist, $7 \alpha-(7-((4,4,5,5,5-$ Pentafluoropentyl)-sulfiny)nonyl)estra-1,3,5(10)-triene$3,17 \beta$-diol (ICI 182,780 , ICI) was purchased from AstraZeneca (Brazil). ICI was dissolved in DMSO at a concentration of $10 \mathrm{mM}$ and stored at $4{ }^{\circ} \mathrm{C}$ in a freezer (as stock solutions). The BMSCs were treated with ICI at $1 \mu \mathrm{M}$ for $48 \mathrm{~h}$.

\section{Cell Counting Kit-8 (CCK-8) assay}

BMSCs were plated at a density of $1 \times 10^{4} /$ well into 96 well plates (Corning, USA). The number of viable cells was analyzed by performing CCK- 8 assay according to the manufacturer's instructions (Tongren, Japan). Lastly, the optical density (OD) was measured at $450 \mathrm{~nm}$ using a microplate reader (TECAN, Switzerland).

\section{Colony formation assay}

To assess the capacity of proliferation, the BMSCs were evenly seeded in six-well plates with $2 \mathrm{~mL}$ culture medium. The medium was refreshed every 3 days. After culturing for 14 days, the cells were washed with phosphate buffered saline (PBS) (Beyotime, China), fixed in 4\% paraformaldehyde (Solarbio, China), and stained with $1 \%$ crystal violet staining solution (Biosharp, China) for $15 \mathrm{~min}$. Subsequently, the cells were rinsed with double distilled water three times. Finally, pictures were taken under an inverted microscope (Nikon, Japan).

\section{Scratch wound healing assay}

The cells were plated at a density of $2 \times 10^{5}$ cells/well in the six-well plates (Corning, USA). After reaching approximately $90 \%$ confluence, scratch wounds were made across each well using a sterile plastic $100-\mu \mathrm{L}$ micropipette tip. After washing with PBS, the images of each scratch were captured, and ImageJ software (NIH, USA) was applied to assess the migration ability by analyzing the residual fractional wound area.

\section{Ribonucleic acid (RNA) isolation and quantitative real- time polymerase chain reaction ( $q R T-P C R)$ assay}

The total RNAs of BMSCs were isolated by using TRIzol reagent (Life Technologies, USA) according to the manufacturer's instructions. Next, a reverse transcription assay was performed using $500 \mathrm{ng}$ total RNAs and a complementary deoxyribonucleic acid (cDNA) synthesis kit (ABI, USA). A qRT-PCR assay was performed using SYBRGreen qPCR Master Mix (Roche, Switzerland). The amplification reactions, containing specific primers and SYBRGreen qPCR Master Mix, were set up in $20-\mu \mathrm{L}$ reaction volumes, and $1 \mu \mathrm{L}$ of $\mathrm{cDNA}$ was added to each amplification reaction. The expression levels of target genes were detected by using the comparative cycle threshold method and normalizing against the expression level of $\beta$-actin. The sequences of the primers used for qRT-PCR analysis were listed in Table 1.

\section{Osteogenic differentiation}

For adipogenic differentiation, BMSCs were seeded in 24well plates (Corning, USA) at a density of $5 \times 10^{5}$ cells/well, and the cells were cultured in osteogenic-inducing medium (Cyagen, USA). The osteogenic-inducing medium was changed every 3 days. After 21 days, the cells were used for further analysis. 
Page 4 of 14

Table 1 The sequence of primers used for qRT-PCR analysis

\begin{tabular}{|c|c|}
\hline Gene (mouse) & Primer sequence ( $5^{\prime}$ to $\left.3^{\prime}\right)$ \\
\hline \multirow[t]{2}{*}{ Nanog } & F: TCTTCCTGGTCCCCACAGTIT \\
\hline & R: GCAAGAATAGTTCTCGGGATGAA \\
\hline \multirow[t]{2}{*}{ Oct4 } & F: CACCATCTGTCGCTTCGAGG \\
\hline & R: AGGGTCTCCGATTTGCATATCT \\
\hline \multirow[t]{2}{*}{ Sox2 } & F: GCGGAGTGGAAACTITTGTCC \\
\hline & R: GGGAAGCGTGTACTTATCCTTCT \\
\hline \multirow[t]{2}{*}{ Runx2 } & F: ACTTCCTGTGCTCCGTGCTG \\
\hline & R: TCGTTGAACCTGGCTACTTGG \\
\hline \multirow[t]{2}{*}{ Osterix } & F: ACCAGGTCCAGGCAACAC \\
\hline & R: GCAAAGTCAGATGGGTAAGTAG \\
\hline \multirow[t]{2}{*}{$\mathrm{OCN}$} & F: AAGCCTTCATGTCCAAGCAG \\
\hline & R: TCCGCTAGCTCGTCACAATT \\
\hline \multirow[t]{2}{*}{ Pparg } & F: GACCACTCGCATTCCTIT \\
\hline & R: CCACAGACTCGGCACTCA \\
\hline \multirow[t]{2}{*}{ Fabp4 } & F: AAATCACCGCAGACGACA \\
\hline & R: CACATTCCACCACCAGCT \\
\hline \multirow[t]{2}{*}{$L P L$} & F: GGGAGTTTGGCTCCAGAGTTT \\
\hline & R: TGTGTCTTCAGGGGTCCTTAG \\
\hline \multirow[t]{2}{*}{$\beta$-actin } & F: CTGTCCCTGTATGCCTCTG \\
\hline & R: TGATGTCACGCACGATTT \\
\hline
\end{tabular}

\section{Alizarin red $S$ (ARS) staining}

After 21 days of osteogenic differentiation, ARS staining was performed to analyze the secreted osteocalcin levels of BMSCs. Next, the cells were stained with ARS staining solution (Cyagen, USA) to evaluate the cell matrix mineralization. To quantify mineralization, ARS was extracted in $10 \%$ cetylpyridinium chloride and detected at $570 \mathrm{~nm}$. An inverted microscope (Nikon, Japan) was used for imaging.

\section{Alkaline phosphatase (ALP) staining}

After 21 days of osteogenic differentiation, ALP staining was applied to assess the osteoblastic mineralization of BMSCs. The ALP activity of cell lysates was then detected by spectrophotometric measurement of p-nitrophenol release using an enzymatic colorimetric ALP kit (Roche, Switzerland). The mineralized nodules were quantified by a microplate reader at $420 \mathrm{~nm}$.
Hua et al. Psoralidin prevents caffeine-induced damage of BMSCs

\section{Adipogenic differentiation}

For adipogenic differentiation, BMSCs were seeded in 24well plates (Corning, USA) at a density of $5 \times 10^{5}$ cells/well, and the cells were cultured in adipogenic-inducing medium (Cyagen, USA). The adipogenic-inducing medium was replaced by fresh medium every 4 days. After 24 days, the cells were used for further analysis.

\section{Oil red $O(O R O)$ staining}

After 24 days of adipogenic differentiation, ORO staining was applied to examine the adipogenesis as previously described (20). The accumulation of oil droplets was regarded as an indicator of adipogenesis in BMSCs. Pictures of the positive area were captured using a light microscope.

\section{Animal experiments}

All protocols and experiments involving the use of animals were approved by the Ethics Committee of the First Affiliated Hospital of Harbin Medical University (2021036) and were performed according to the National Institutes of Health Guide for the Care and Use of Laboratory Animals (NIH Publications no. 85-23, revised 1996). All animals were purchased from Beijing Vital River Laboratory Animal Technology, China. Sixty 8-week-old female C57BL/6J mice (approximately 18-20 g) were maintained under specific pathogen-free conditions $\left(22{ }^{\circ} \mathrm{C}, 50-55 \%\right.$ humidity, and $12 \mathrm{~h}$ light/dark cycles), and food and water were easily accessible. All mice were randomly divided into three groups ( $n=20$ in each group): control group, caffeinetreated group, and caffeine/PL-treated group. Caffeine in the caffeine-treated group was used at a dose of $20 \mathrm{mg} / \mathrm{kg}$ by intragastric administration once daily for 4 weeks. Highdose caffeine was applied to establish the caffeine-induced osteoporotic mouse models. PL in the caffeine/PL-treated group was quantitatively administered $(10 \mathrm{mg} / \mathrm{kg})$ via the caudal vein for 28 consecutive days. Mice in the control group were quantitatively administered normal saline. After the last administration, the mice from the three groups were obtained and used for further analysis.

\section{Micro-computed tomography $(\mu C T)$ scanning}

The femurs of mice from different groups were detached after sacrifice and fixed in $4 \%$ paraformaldehyde for $24 \mathrm{~h}$. Next, the femurs were isolated and scanned using $\mu \mathrm{CT}$ 
(SCANCO, Switzerland) scanning at a voxel resolution of $10.5 \mu \mathrm{m}$. Three-dimensional morphological parameters, including bone volume/tissue volume (BV/TV), trabecular number (Tb.N), bone mineral density (BMD), and trabecular separation (Tb.Sp) were obtained and analyzed.

\section{Statistical analysis}

All quantitative data were presented as mean \pm standard deviation (SD). All experiments were repeated at least three times, and representative experiments were shown. Statistical comparisons were performed by using Student's $t$-test or one-way analysis of variance by GraphPad Prism 7 software (GraphPad, CA). $\mathrm{P}<0.05$ was regarded as significant.

\section{Results}

Caffeine suppressed the viability, proliferation, migration, and pluripotency of BMSCs

In order to assess the roles of caffeine $(1,3,7$ -trimethylxanthine) in the biological functions of BMSCs, BMSCs were incubated with caffeine (Figure 1A). To establish the appropriate dose, the BMSCs were treated with different concentrations of caffeine $(0.01,0.05,0.1,0.5$, and $1.0 \mathrm{mM}$ ) for $48 \mathrm{~h}$. A CCK-8 assay was performed to detect the roles of caffeine in the cell viability of BMSCs. As shown in Figure $1 B$, the exposure to different concentrations of caffeine for $48 \mathrm{~h}$ resulted in the decreased viability of BMSCs in a dose-dependent manner, revealing that the various concentrations of caffeine $(0.01,0.05,0.1,0.5,1.0 \mathrm{mM})$ inhibited the growth of BMSCs (Figure 1B).

In order to assess the effects of caffeine on the proliferation potential of BMSCs, a colony formation assay was applied. The results showed that with the concentration of caffeine increasing from 0.01 to $1.0 \mathrm{mM}$, the number of colonies was gradually reduced (Figure 1C,1D).

Furthermore, to examine the effects of caffeine on the migration of BMSCs, a scratch wound healing assay was performed. The results demonstrated that cells exposed to caffeine exhibited significantly decreased migration ability, indicating that different concentrations of caffeine $(0.01$, $0.05,0.1,0.5,1.0 \mathrm{mM}$ ) decreased the cell migration ability of BMSCs, especially 0.5 and $1.0 \mathrm{mM}$ (Figure $1 E, 1 F$ ).

BMSCs expressed pluripotency-associated transcription factors, including Nanog, Sox2, and Oct4, and qRT-PCR analysis was applied to determine the expression levels of these genes. As presented in Figure 1G, the expression of Nanog, Sox2, and Oct4 in BMSCs was decreased in a concentration-dependent manner after caffeine treatment (Figure 1G). Therefore, the above results indicated that different concentrations of caffeine could damage the viability, proliferation, migration, and pluripotency of BMSCs.

\section{Caffeine blocked the osteogenic differentiation and promoted the adipogenic differentiation of BMSCs}

In order to analyze whether the multilineage differentiation potentials of BMSCs were affected by caffeine treatment, BMSCs were treated with caffeine and then induced into osteoblasts or adipocytes. BMSCs were first exposed to different concentrations of caffeine $(0.01,0.05,0.1,0.5$, and $1.0 \mathrm{mM}$ ) and then cultured in the osteogenic-inducing medium. After 21 days, mineralized nodules were formed in BMSCs. ALP staining, ARS staining, and qRT-PCR analysis were used to evaluate the osteogenic differentiation of BMSCs. ARS staining indicated that different concentrations of caffeine, particularly at $0.5 \mathrm{mM}$ and $1.0 \mathrm{mM}$, markedly decreased the mineralized areas, suggesting that caffeine inhibited the differentiation of BMSCs into osteoblast lineage (Figure 2A,2B). According to the results of ALP staining, we observed that exposure to caffeine at $0.01,0.05,0.1,0.5$, and $1.0 \mathrm{mM}$ resulted in significantly fewer mineralized nodules, which indicated that caffeine suppressed the formation of mineralized nodules in BMSCs (Figure 2C,2D). The change in mineralization of the BMSCs was consistent with the expression levels of osteoblast-related genes, including runt related transcription factor 2 (Runx2), Osterix, osteocalcin (OCN). The results of the qRT-PCR analysis demonstrated that caffeine treatment induced gradually decreased expression levels of these genes in BMSCs at concentrations ranging from 0.01 to $1.0 \mathrm{mM}$, and especially at 0.5 and $1.0 \mathrm{mM}$ (Figure $2 E$ ).

Similarly, we also confirmed the effect of caffeine on the adipogenesis of BMSCs by ORO staining and qRTPCR analysis. BMSCs were first exposed to different concentrations of caffeine $(0.01,0.05,0.1,0.5$, and $1.0 \mathrm{mM})$ and then cultured in the adipogenic-inducing medium. After 24 days, oil droplets were observed in the BMSCs. As shown in Figure $2 F$ and $2 G$, after exposing the BMSCs to caffeine from 0.01 to $1.0 \mathrm{mM}$, the cells exhibited more lipid droplets compared to the control group, which indicated that caffeine promoted the adipogenesis of BMSCs (Figure 2F,2G). Next, the expression levels of adipocyte- 
A<smiles>Cn1c(=O)c2c(ncn2C)n(C)c1=O</smiles>

Caffeine
(1,3,7-trimethylxanthine)
$E$
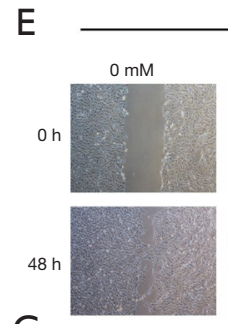

G

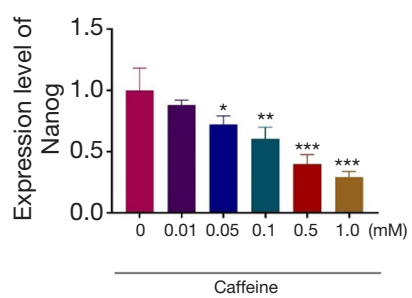

B

C

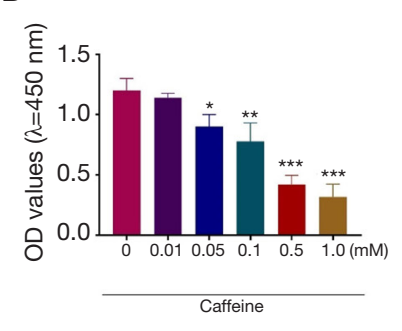

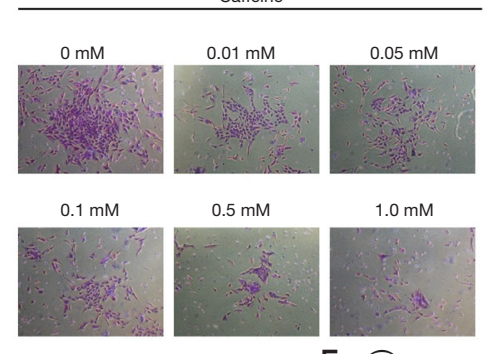

D

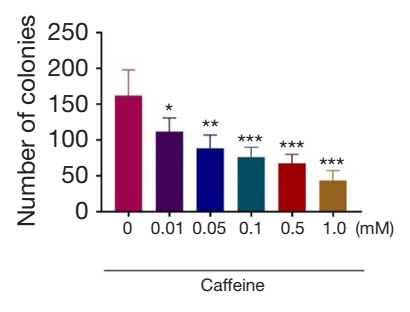

Figure 1 The inhibitory effects of caffeine on the viability, proliferation, migration, and pluripotency of BMSCs. (A) Chemical structures of caffeine. (B) The CCK-8 assay was used to assess the cell viability of BMSCs after treatment with caffeine at $0.01,0.05,0.1,0.5$, and 1.0 $\mathrm{mM}$ for $48 \mathrm{~h}$. (C) The colony formation assay was performed to determine the proliferative ability of BMSCs treated with $0.01,0.05,0.1$, 0.5 , and $1.0 \mathrm{mM}$ caffeine (100x). (D) The statistical results of the colony formation assay. (E) The migration of BMSCs in the presence of caffeine was determined by the wound healing assay (100x). (F) Quantitative analysis of the wound healing assay. (G) QRT-PCR analysis was applied to measure the mRNA expression levels of pluripotency-associated transcription factors, including Nanog, Sox2 and Oct $4 . \mathrm{n}=3$. *, $\mathrm{P}<0.05 ;{ }^{* *}, \mathrm{P}<0.01 ;{ }^{* *}, \mathrm{P}<0.001$. BMSCs, bone marrow mesenchymal stem cells.

related genes, including peroxisome proliferator-activated receptor gamma (Pparg), fatty acid binding protein 4 (Fabp4), and lipoprotein lipase (LPL), were further analyzed. As shown in Figure $2 \mathrm{H}$, caffeine induced the expression of Pparg, Fabp4, and LPL, especially at concentrations of 0.5 and $1.0 \mathrm{mM}$ (Figure $2 \mathrm{H}$ ). The above results suggested that caffeine inhibited the osteogenic differentiation, but promoted the adipogenic differentiation of BMSCs.

Notably, 0.5 and $1 \mathrm{mM}$ caffeine significantly inhibited the cell viability, proliferation, migration, pluripotency, and osteogenic differentiation, but promoted the adipogenic differentiation of BMSCs. Considering of toxicity of caffeine, 48 -h exposure to $0.5 \mathrm{mM}$ caffeine was selected as the standard pretreatment in subsequent experiments.

\section{The effects of PL on caffeine-induced dysfunctions of BMSCs}

To test whether PL could reverse the caffeine-induced aberrant biological functions of BMSCs, BMSCs were treated with caffeine at $0.5 \mathrm{mM}$ and further exposed to different concentrations of PL $(0.01,0.1,1.0$, and $10 \mu \mathrm{M})$ for $48 \mathrm{~h}$ (Figure $3 A$ ). Next, CCK-8 assay, colony formation assay, wound healing assay, and qRT-PCR analysis were performed. As shown in Figure 3B, caffeine at $0.5 \mathrm{mM}$ dramatically inhibited the cell viability of BMSCs, while it was elevated by PL (0.01, 0.1, 1, and $10 \mu \mathrm{M}$ ) (Figure 3B). Furthermore, BMSCs pretreated with caffeine at $0.5 \mathrm{mM}$ exhibited reduced colony formation, and the numbers of colonies were increased by different concentrations of PL in dose-dependent manners, indicating that caffeine at 


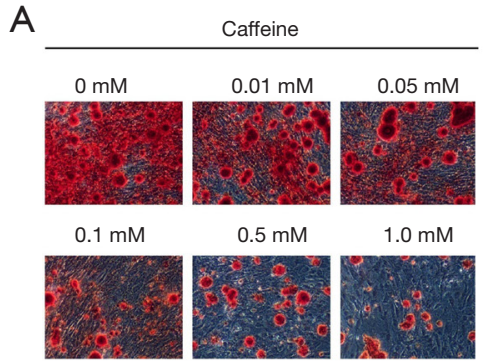

$\mathrm{E}$

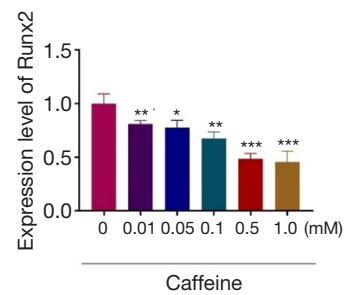

G

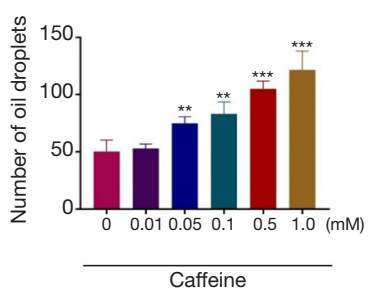

B

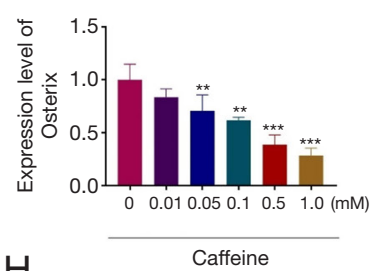

$\mathrm{H}$

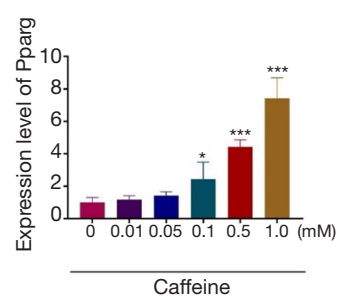

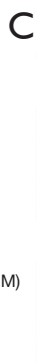

C
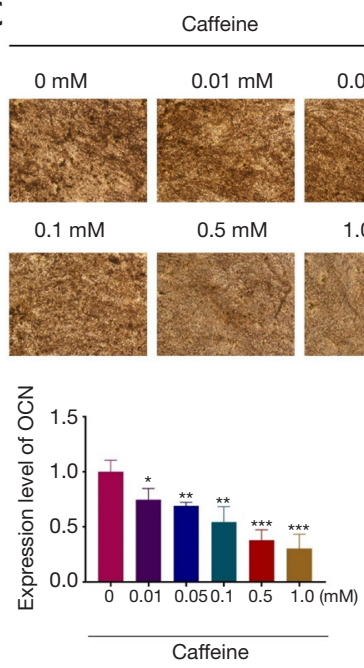

$\mathrm{F}$
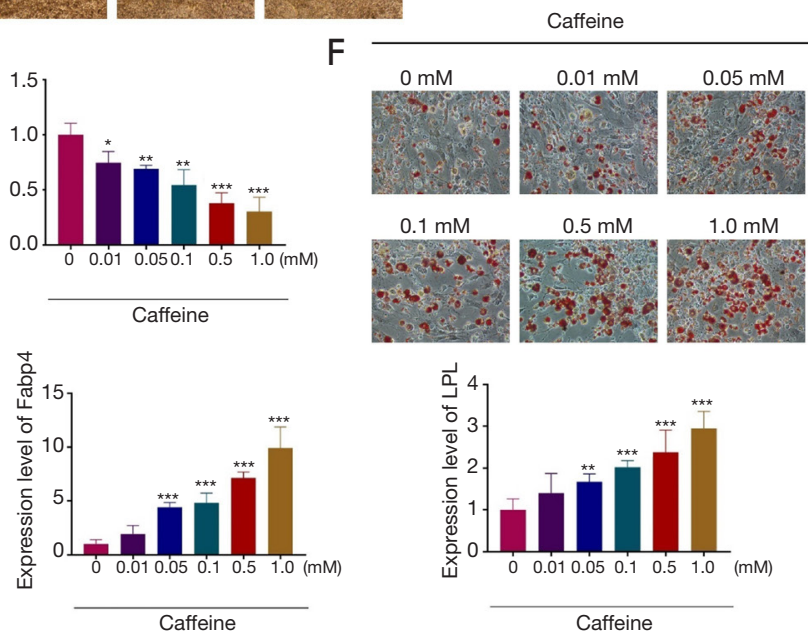

D
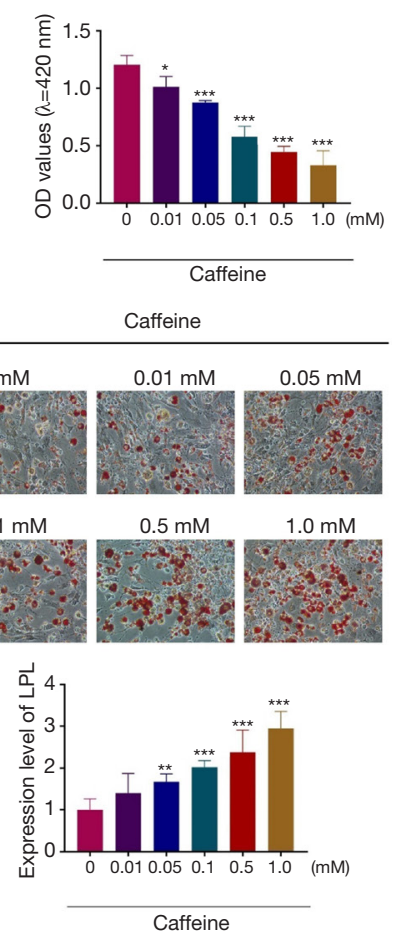

Figure 2 The roles of caffeine in the differentiation of BMSCs. (A) ARS staining of BMSCs cultured in different concentrations of caffeine $(0.01,0.05,0.1,0.5$, and $1.0 \mathrm{mM})$ after osteogenic differentiation for 21 days (100x). (B) Quantitative analysis of ARS staining; (C) ALP staining of BMSCs treated with different concentrations of caffeine $(0.01,0.05,0.1,0.5$, and $1.0 \mathrm{mM})$ after osteogenic differentiation for 21 days (100x). (D) Quantitative analysis of ALP staining; (E) QRT-PCR was used to evaluate the gene expression of osteogenic markers in BMSCs after treatment with caffeine. (F,G) After adipogenic induction for 24 days, the droplet formation in BMSCs after caffeine treatment was analyzed by ORO staining (200×). (H) QRT-PCR analysis of Pparg, Fabp4, and LPL expression in BMSCs pretreated with caffeine for 48 h. $\mathrm{n}=3$. *, $\mathrm{P}<0.05$; **, $\mathrm{P}<0.01$; ***, $\mathrm{P}<0.001$. BMSCs, bone marrow mesenchymal stem cells; QRT-PCR, quantitative real-time polymerase chain reaction.

$0.5 \mathrm{mM}$ suppressed the proliferative ability of BMSCs, which could be reversed by PL (Figure 3C,3D). Also, compared with control group, caffeine treatment induced significantly decreased migration ability of BMSCs, shown by the wound healing assay (Figure $3 E, 3 F$ ). However, this was reversed by PL at $0.01,0.1,1$, and $10 \mu M$ in a concentration-dependent manner (Figure 3E, $3 F$ ).

The mRNA expression levels of Nanog, Sox2, and Oct4 were markedly lower in BMSCs exposed to caffeine than in the control group, which could be increased in the presence of PL in a concentration-dependent manner (Figure 3G). Taken together, these data suggested that the caffeineinduced damage to BMSCs could be reversed by PL treatment.

\section{The roles of $P L$ in caffeine-caused aberrant differentiation of BMSCs}

In order to clarify the effects of PL $(0.01,0.1,1,10 \mu \mathrm{M})$ on the differentiation of BMSCs pretreated with caffeine, osteogenesis and adipogenesis were evaluated. As shown in Figure 4A, $0.5 \mathrm{mM}$ caffeine could significantly inhibit the mineralization formation of BMSCs, revealed by ARS staining (Figure $4 A, 4 B$ ). However, treatment with PL at $0.01,0.1,1$, and $10 \mu \mathrm{M}$ could effectively reverse the reduced mineralization caused by caffeine (Figure $4 A, 4 B$ ). Also, BMSCs exposed to $0.5 \mathrm{mM}$ caffeine exhibited less mineralized nodules, which were increased by different concentrations of PL (Figure 4C,4D). After osteogenesis 

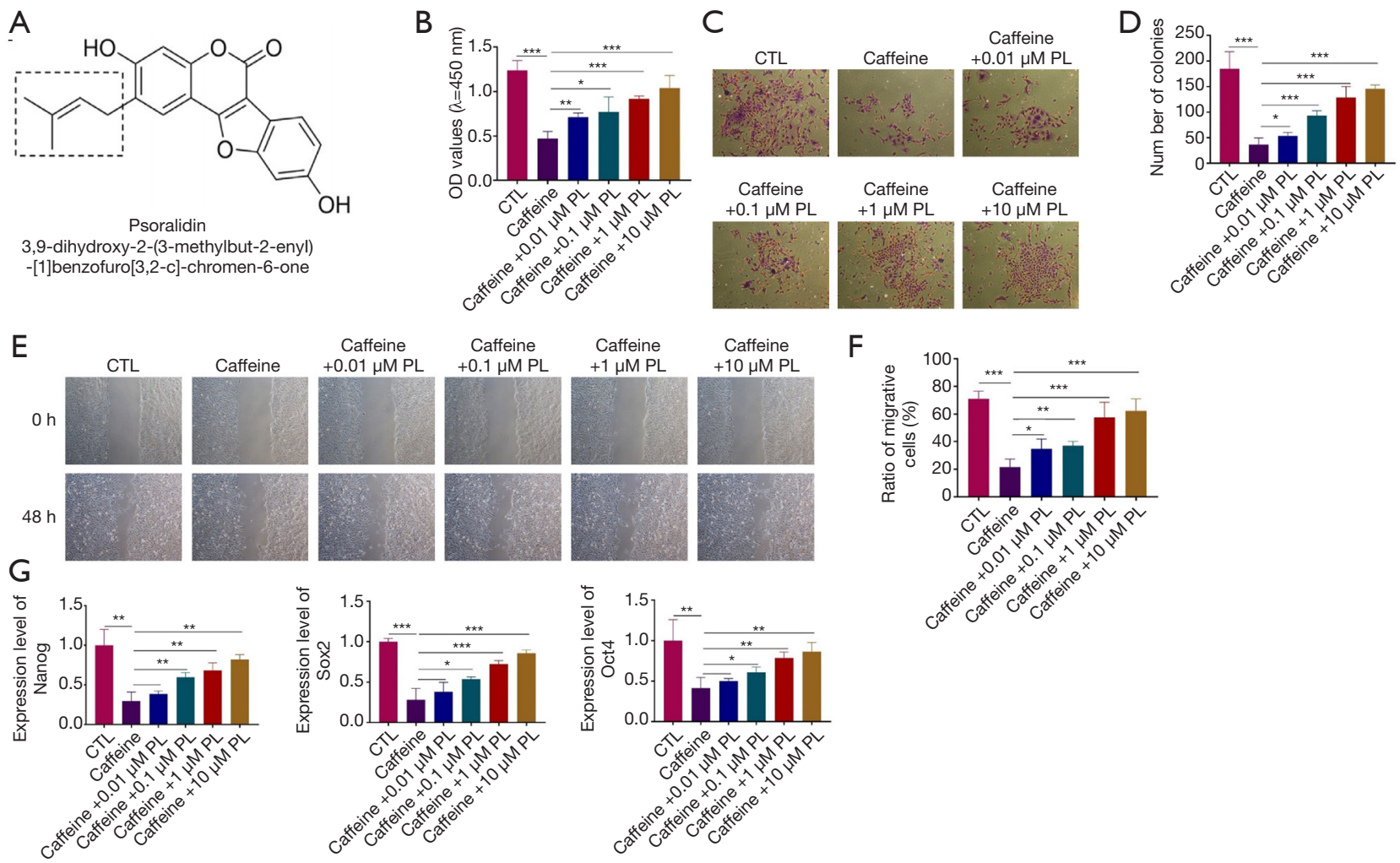

Figure 3 The effects of PL on the caffeine-induced dysfunctions of BMSCs. (A) Chemical structures of PL. (B) BMSCs were treated with PL $(0.01,0.1,1.0$, and $10 \mu \mathrm{M})$ in the presence of caffeine for $48 \mathrm{~h}$ and the cell viability was determined by CCK- 8 assay. (C) The colony formation assay was performed after different concentrations of PL and $0.5 \mathrm{mM}$ caffeine treatment for $48 \mathrm{~h}$ (100x). (D) Statistical results of the colony formation assay. (E) The migration ability in BMSCs after PL treatment for $48 \mathrm{~h}$ in the presence of caffeine (100x). (F) Quantitative analysis of the wound healing assay; $(G)$ The expression of Nanog, Sox2, Oct4 in BMSCs after different concentrations of PL and $0.5 \mathrm{mM}$ caffeine treatment for $48 \mathrm{~h} . \mathrm{n}=3 .{ }^{*}, \mathrm{P}<0.05 ;{ }^{* *}, \mathrm{P}<0.01 ;{ }^{* * *}, \mathrm{P}<0.001$. PL, psoralidin; BMSCs, bone marrow mesenchymal stem cells.

and mineralization of BMSCs, we measured the mRNA expression levels of osteogenic markers (Runx2, Osterix, and OCN) at day 21 after osteogenic stimulation. The results of qRT-PCR analysis revealed that compared to the control group, the expression levels of Runx2, Osterix, and OCN were lower in the caffeine-treated group (Figure 4E). Interestingly, the reduced expression levels of Runx2, Osterix, and OCN were elevated by PL in a dosedependent manner (Figure 4E).

Subsequently, we further investigated the roles of PL in the adipogenic differentiation of caffeine-treated BMSCs. After adipogenic differentiation of BMSCs for 24 days, ORO staining displayed that many lipid droplets were observed in BMSCs in the caffeine-treated group, while PL $(0.01,0.1,1,10 \mu \mathrm{M})$ inhibited the accumulation of oil droplets (Figure $4 F, 4 G$ ). Consistent with the staining results, the expression levels of adipogenic markers (Pparg,
Fabp4 and LPL) were significantly higher in the presence of caffeine than in the control group, whereas the addition of PL markedly inhibited the mRNA expression levels of these genes (Figure 4H). These results confirmed that the inhibitory effects of caffeine on osteogenesis and its accelerative effects on adipogenesis of BMSCs were observably suppressed by PL treatment.

\section{$P L$ prevented bone loss of the caffeine-induced osteoporotic mice}

Sixty 8-week-old female were randomly divided into three groups: control group, caffeine-treated group, and caffeine/PL-treated group. Osteoporotic mouse models were constructed by intragastric administration at a dose of $20 \mathrm{mg} / \mathrm{kg}$ once daily for 4 weeks to observe the caffeine-induced damage and bone loss in mice. PL 
A

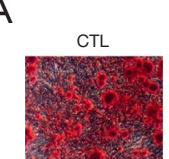

Caffeine +

$0.1 \mu \mathrm{M} \mathrm{PL}$

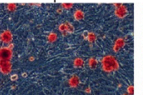

E
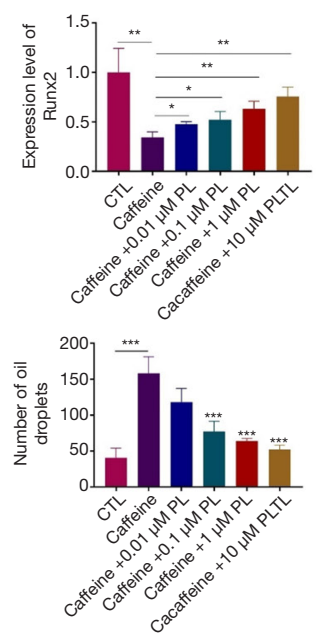

B

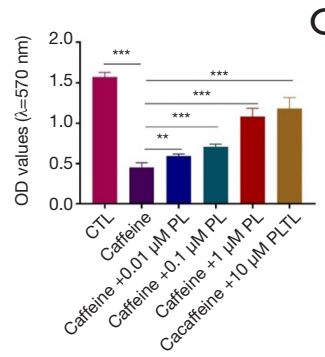

C
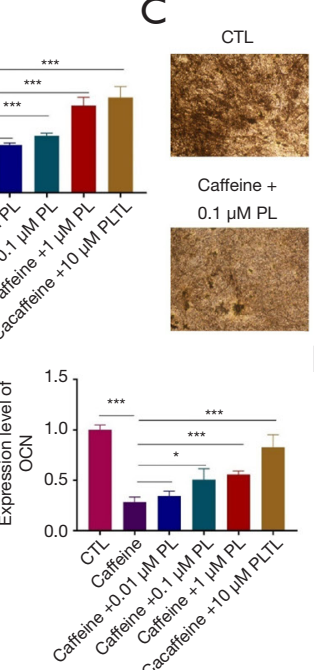

$\mathrm{H}$
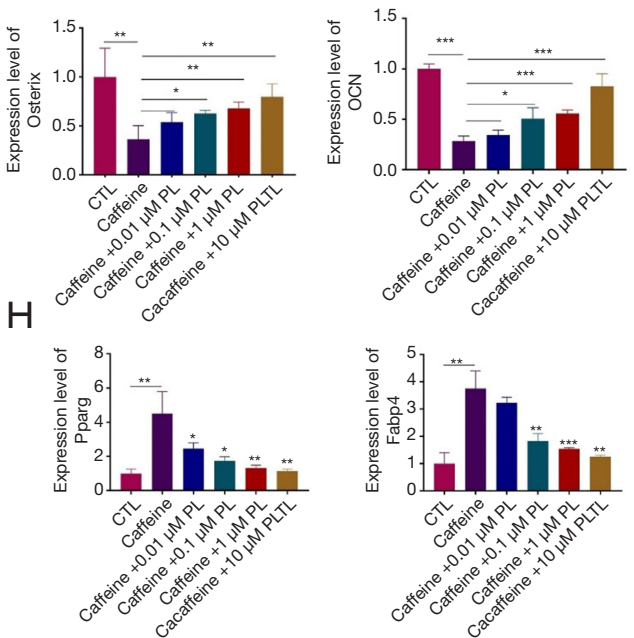

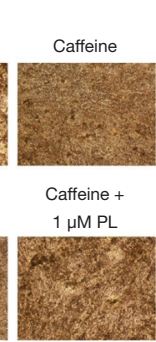

$\mathrm{F}$
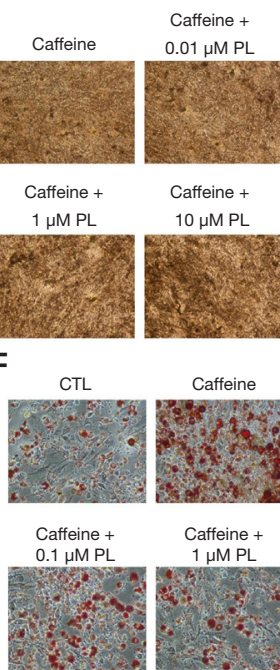

Caffeine

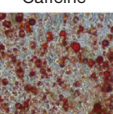

Caffeine +
$1 \mu \mathrm{M}$ PL

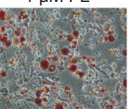

Caffeine
$10 \mu \mathrm{M} \mathrm{P}$

$\mathrm{D}$

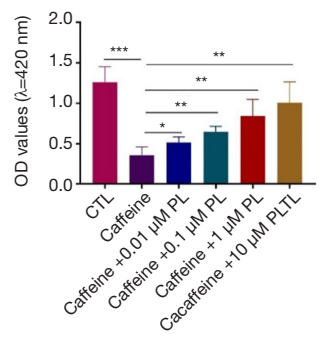

Caffeine +

$0.01 \mu \mathrm{MPL}$
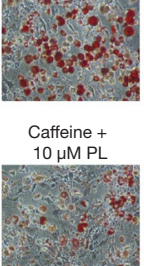

Figure 4 The roles of PL in caffeine-induced aberrant differentiations of BMSCs. (A) ARS staining of calcium depositions in BMSCs after PL and caffeine treatment (100x). (B) Quantitative analysis of ARS staining. (C) The dose-dependent effects of PL on the osteogenesis of BMSCs treated with caffeine (100x). (D) Quantitative analysis of ALP staining. (E) The expression levels of Runx2, Osterix, and OCN in BMSCs after treatment with caffeine and PL were measured by qRT-PCR analysis. (F) The oil droplets in the presence of caffeine and PL were stained using ORO staining (200x). (G) Quantitative analysis of ORO staining. (H) The expression of Pparg, Fabp4, and LPL in BMSCs subjected to caffeine and PL was determined by qRT-PCR assay. $\mathrm{n}=3$. ${ }^{*}, \mathrm{P}<0.05 ;{ }^{* *}, \mathrm{P}<0.01$; ${ }^{* * *}, \mathrm{P}<0.001$. PL, psoralidin; BMSCs, bone marrow mesenchymal stem cells.

was quantitatively administered at a dose of $10 \mathrm{mg} / \mathrm{kg}$ via the caudal vein for 28 consecutive days to determine the protective effects of PL on caffeine-caused injury in mice. After the last administration, the bone tissues from different groups were obtained and used to detect the bone microarchitecture index, including BV/TV, Tb.N, BMD, and Tb.Sp. $\mu \mathrm{CT}$ analysis showed that compared to the control group, BV/TV, Tb.N, and BMD of mice from the caffeine-treated group was markedly lower, but the Tb.Sp was significantly higher, indicating that the caffeine-induced osteoporotic mouse models were successfully established (Figure $5 A$ ). Also, mice from the caffeine/PL-treated group exhibited increased BV/TV, Tb.N, and BMD, and reduced Tb.Sp, indicating that PL treatment could prevent the caffeine-induced bone loss of osteoporotic mice (Figure 5A).

Next, to determine the effects of PL on the osteogenic differentiation of BMSCs from caffeine-induced osteoporotic mice, BMSCs from the control group, caffeine-treated group, caffeine/PL-treated group were isolated and induced into osteoblasts for 21 days. ARS staining revealed that compared with control group, the areas and number of mineralized nodules were decreased in the BMSCs from caffeine-treated group, suggesting that caffeine treatment inhibited the differentiation of BMSCs into osteoblast lineage in caffeine-related osteoporotic mice (Figure 5B,5C). As shown in Figure 5B and 5C, BMSCs from the caffeine/PL-treated group showed an increase in the quantity of calcium deposition compared with caffeinetreated group, which showed that PL treatment could effectively prevent the decreased osteogenic differentiation of BMSCs from caffeine-caused osteoporotic mice (Figure 5B,5C). ALP staining confirmed that the BMSCs 


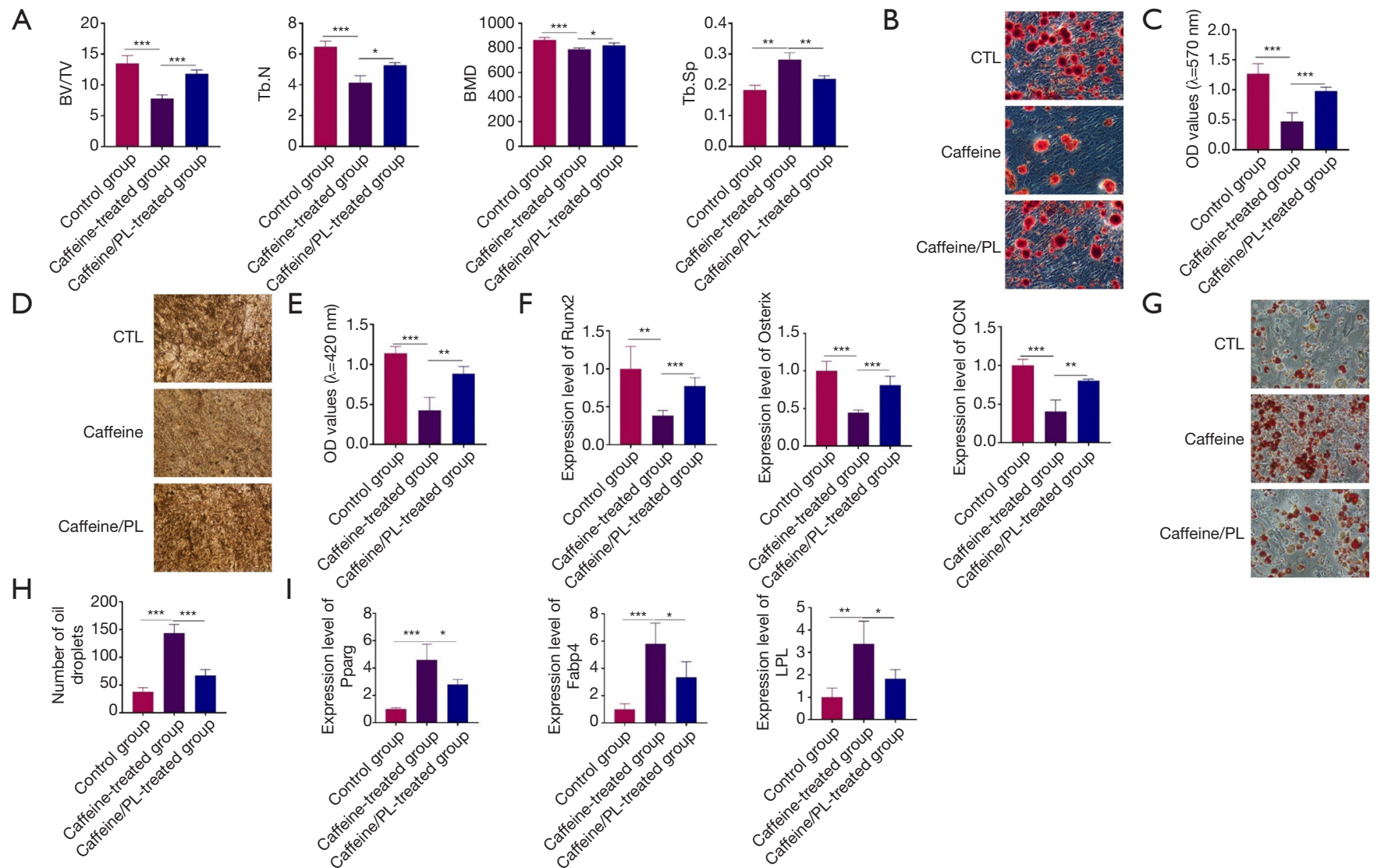

Figure 5 PL reversed the bone loss of caffeine-induced osteoporotic mice. (A) $\mu \mathrm{CT}$ analysis of the bone microstructure of mice. (B) BMSCs isolated from mice were cultured in the osteo-inductive medium for 21 days and were stained by ARS staining (100x). (C) Quantitative analysis of ARS staining. (D) BMSCs collected from mice were maintained in the osteogenic-inducing medium for 21 days and were stained by ALP staining (100x). (E) Quantitative analysis of ALP staining. (F) The cells cultured in the osteo-inductive medium for 21 days were collected and analyzed for the expression of Runx2, Osterix, and OCN using qRT-PCR analysis. (G) BMSCs cultured in adipo-inductive medium for 24 days were collected and analyzed by ORO staining (200x). (H) Quantitative analysis of ORO staining; (I) BMSCs from mice were induced into adipocytes and applied for the expression of Pparg, Fabp4, and LPL. $\mathrm{n}=3$. * $\mathrm{P}<0.05$; **, $\mathrm{P}<0.01$; ***, $\mathrm{P}<0.001$. BV/TV, bone volume/tissue volume; Tb.N, trabecular number; BMD, bone mineral density; Tb.Sp, trabecular separation

isolated from the caffeine-induced osteoporotic mice exhibited lower osteogenic potentials compared to the control group (Figure 5D,E). ALP staining also showed that the decreased osteogenesis of BMSCs in the caffeinetreated group could be restrained by PL treatment (Figure 5D, 5E). Consistent with the staining results, the expression levels of the osteoblast-specific genes were markedly reduced in BMSCs from the caffeine-related osteoporotic mice compared to the control group, and were elevated by PL treatment (Figure $5 F$ ).

To further identify the effects of PL on the adipogenic differentiation of BMSCs from caffeine-induced osteoporotic mice, BMSCs were isolated from the control, caffeine-treated, and caffeine/PL-treated groups, and cultured in adipogenic-inducing medium for 24 days. ORO staining showed that compared with control group, caffeine increased the number of lipid droplets in the caffeinetreated group (Figure 5G,5H). We also observed that the stronger lipid deposits caused by caffeine administration could be reduced by PL, confirming that the promotive effects of caffeine on the adipogenesis of BMSCs in vivo could be offset by PL treatment (Figure 5G,5H). QRTPCR analysis validated that excessive caffeine increased the adipogenic markers compared with the control in mice, and were inhibited by PL treatment (Figure 5I). These observations indicated that excessive caffeine could 
A

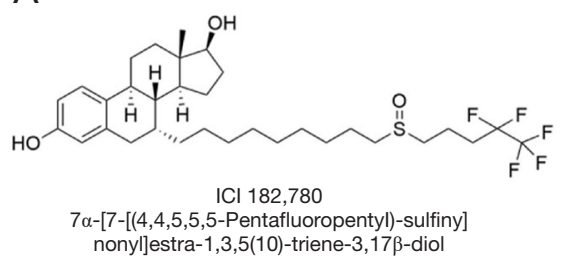

E

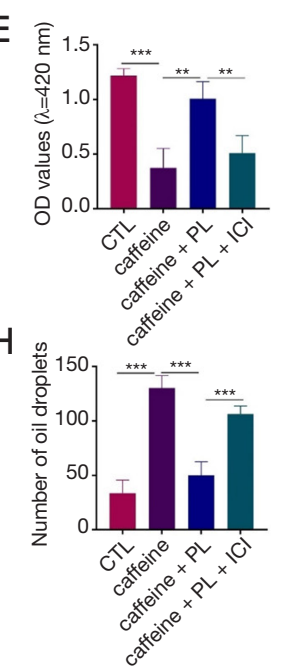

$\mathrm{F}$
B
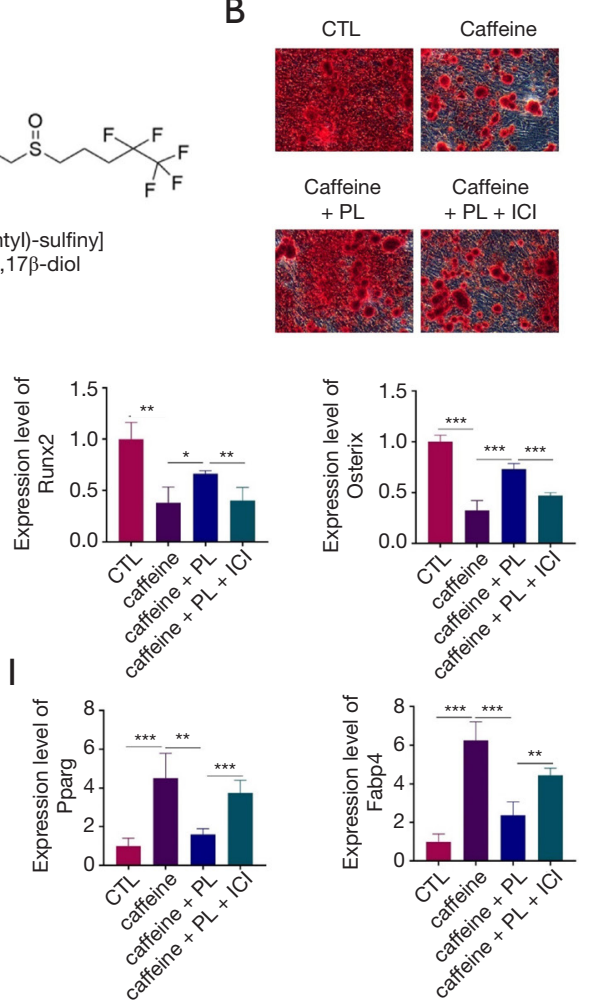

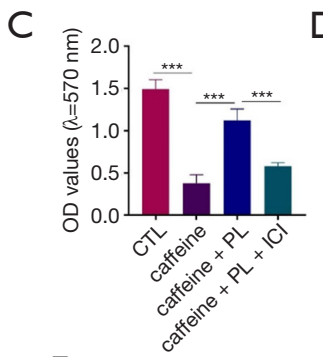

G

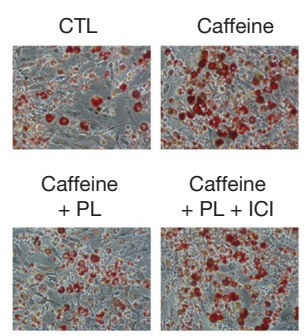

Figure 6 PL reversed the effects of caffeine in BMSCs via the ER pathway. (A) Chemical structures of ICI. (B) ARS staining was performed to detect the osteogenesis of BMSCs in the presence of caffeine, caffeine + PL, and caffeine + PL + ICI (100x). (C) Quantitative analysis of ARS staining. (D) ALP staining was used to measure the osteogenesis of BMSCs in the presence of caffeine, caffeine + PL, and caffeine + PL + ICI (100x); (E) Quantitative analysis of ALP staining. (F) QRT-PCR analysis was applied to assess the expression of Runx2, Osterix, and OCN in BMSCs after treatment with caffeine, caffeine + PL, and caffeine + PL + ICI. (G) ORO staining was conducted to determine the oil droplets of BMSCs in the presence of caffeine, caffeine + PL, and caffeine + PL + ICI (200x). (H) Quantitative analysis of ORO staining; (I) QRT-PCR analysis was applied to analyze the expression of Pparg, Fabp4, and LPL in BMSCs after caffeine, caffeine + PL, and caffeine + $\mathrm{PL}+\mathrm{ICI}$ treatment. $\mathrm{n}=3 .{ }^{*}, \mathrm{P}<0.05 ;{ }^{* *}, \mathrm{P}<0.01 ;{ }^{* * *}, \mathrm{P}<0.001$.

induce bone loss and OP by inhibiting the osteogenesis but improving the adipogenesis of BMSCs, which could be prevented by PL treatment.

\section{PL inbibited the caffeine-caused aberrant differentiation of BMSCs via the classical ER patbway}

To further examine whether the classical ER pathway was involved in mediating the protective effects of $\mathrm{PL}$ on the caffeine-induced abnormal differentiation in BMSCs, ICI 182,780 (ICI), known as the specific antagonist of ER, was applied (Figure $6 \mathrm{~A}$ ). The cells were treated with caffeine, caffeine + PL, and caffeine + PL + ICI 182,780, respectively. As shown in Figure $6 B$ and $6 C$, ARS staining revealed that the reduced mineralization nodules (caused by caffeine) were elevated by PL treatment in BMSCs, whereas they could be lowered in the presence of ICI (Figure 6B,6C). ALP staining indicated that caffeine could significantly suppress the osteogenesis of BMSCs, which was inhibited after PL treatment (Figure 6D,6E). Moreover, ICI treatment markedly inhibited the mineralization of BMSCs, which was promoted by PL (Figure 6D,6E). QRT-PCR analysis showed that the expression of osteoblastic markers (Runx2, Osterix, and OCN) was decreased in the cells exposed to caffeine, which could be reversed in the presence of PL (Figure $6 F$ ). Moreover, the roles of PL were abolished in the presence of ICI (Figure 6F).

As shown by ORO staining, after the adipogenesis process of 24 days, caffeine increased the number and areas of droplet formation, which could be blocked by 
PL (Figure 6G,6H). Compared to the caffeine + PLtreated group, the formation and accumulation of lipid droplets were significantly higher in the caffeine + PL + ICI-treated group (Figure 6G,6H). As for the expression of adipogenesis-related genes (Pparg, Fabp4, and LPL), PL completely abolished the promotive effects of caffeine in BMSCs, which could be blocked by ICI treatment (Figure 6I). The data showed that PL treatment could reverse the caffeine-induced aberrant differentiation of BMSCs, which was blocked in the presence of ICI, suggesting that PL reversed the caffeine-induced damage via the ER pathway.

\section{Discussion}

Bone diseases, such as OP, are caused by decreased bone formation and an increased number of adipocytes (21). BMSCs are a type of self-renewing multipotent cell and have been extensively applied in the treatment of multiple musculoskeletal injuries and disorders $(22,23)$. Maintaining skeletal homeostasis depends on the balance between osteogenesis and adipogenesis of BMSCs (24-26). Hence, targeting biological functions and differentiation of BMSCs might be a novel alternative strategy for the treatment of bone diseases, including OP (11). In this study, we demonstrated that caffeine induced inhibitory effects on the cell viability, proliferation, migration, and pluripotency of BMSCs in vitro. Further analysis indicated that excessive caffeine could induce $\mathrm{OP}$ via the suppression of osteogenesis and the promotion of adipogenesis of BMSCs. A previous study suggested that caffeine is correlated with decreased BMD (13). Consistent with previous reports, our results showed that caffeine induced bone loss and OP in mice by regulating the osteogenesis and adipogenesis of BMSCs.

PL, a metabolic product from the seed of Psoralea corylifolia, exerts anti-inflammatory and immunomodulatory functions (27). It is commonly applied in traditional Chinese medicine because of its pharmacological properties, including anti-bacterial, anti-oxidative, radio-preventive, anti-inflammatory, antidepressive, and estrogenic-like roles $(14,28)$. PL also plays some roles in oxidative stress, cell proliferation, apoptosis, and autophagy (14). The present study revealed that PL exerted inhibitory effects on the caffeine-induced injury in BMSCs and bone loss in mice. However, the mechanism of PL on the caffeine-induced injury in BMSCs and bone loss in mice has not yet been investigated. Therefore, we aimed to investigate whether PL could reverse excessive caffeine-caused injury in vitro and in vivo and, if so, to elucidate the underlying mechanisms. Documented studies have demonstrated that PL regulates several extracellular signaling pathways, which include the protein kinase $\mathrm{B} /$ glycogen synthase kinase $3 \beta / \beta$-catenin signaling pathway, the nuclear factor-kappa $\mathrm{B}(\mathrm{NF}-\kappa \mathrm{B})$ and phosphatidylinositol 3 kinase (PI3K)/Akt signaling pathway, and the NF- $\mathrm{kB}$ and B-cell lymphoma-2 (Bcl-2)/ $\mathrm{Bcl}-2$ associated $\mathrm{X}$ protein ( $\mathrm{Bax}$ ) signaling pathway $(16,29,30)$. Previous studies have demonstrated that PL can inhibit the formation of adipocytes by modulating the classical and membrane ER pathways (16). The results of our study indicated that PL treatment could reverse the caffeine-induced dysfunctions and aberrant differentiation of BMSCs, and even the bone loss of mice, via a classical ER pathway. We report for the first time that different concentrations of caffeine could exert a damaging effect on the biological functions and differentiation of BMSCs, and PL treatment could reverse the damage both in vivo and in vitro. We also developed a novel therapeutic method that can be applied in the treatment of excessive caffeineinduced bone loss.

Taken together, our results strongly suggested that PL could play a crucial role in the cell viability, proliferation, migration, pluripotency, and differentiation potentials of BMSCs, and thereby reverse the dysfunctions and aberrant differentiation caused by excessive caffeine. Importantly, PL could prevent the bone loss of caffeine-induced osteoporotic mice by regulating the osteogenesis and adipogenesis of BMSCs. Moreover, PL treatment can effectively promote the recovery from caffeine-induced injury of BMSCs and osteoporotic mice, and the mechanism might be related to the ER pathway.

\section{Acknowledgments}

Funding: This study was supported by the Graduate Innovation Fund of Harbin Medical University (YJSSJCX2019-09HYD) and the Projects of Medical and Health Technology Development Program in Shandong Province (202002040581), Natural Science Foundation of Jiangsu Province (SBK2021042454) and Natural Science Foundation of Heilongjiang Province (LH2021H053).

\section{Footnotes}

Reporting Checklist: The authors have completed the ARRIVE reporting checklist. Available at https://dx.doi. 
org/10.21037/atm-21-3153

Data Sharing Statement: Available at https://dx.doi. org/10.21037/atm-21-3153

Conflicts of Interest: All authors have completed the ICMJE uniform disclosure form (available at https://dx.doi. org/10.21037/atm-21-3153). The authors have no conflicts of interest to declare.

Ethical Statement: The authors are accountable for all aspects of the work in ensuring that questions related to the accuracy or integrity of any part of the work are appropriately investigated and resolved. All protocols and experiments involving the use of animals were approved by the Ethics Committee of the First Affiliated Hospital of Harbin Medical University (2021036) and were performed according to the National Institutes of Health Guide for the Care and Use of Laboratory Animals (NIH Publications no. 85-23, revised 1996).

Open Access Statement: This is an Open Access article distributed in accordance with the Creative Commons Attribution-NonCommercial-NoDerivs 4.0 International License (CC BY-NC-ND 4.0), which permits the noncommercial replication and distribution of the article with the strict proviso that no changes or edits are made and the original work is properly cited (including links to both the formal publication through the relevant DOI and the license). See: https://creativecommons.org/licenses/by-nc-nd/4.0/.

\section{References}

1. Qi M, Zhang L, Ma Y, et al. Autophagy Maintains the Function of Bone Marrow Mesenchymal Stem Cells to Prevent Estrogen Deficiency-Induced Osteoporosis. Theranostics 2017;7:4498-516.

2. Sitati FC, Gichangi P, Obimbo MM, et al. Prevalence of osteoporosis and its associated factors among postmenopausal women in Kiambu County, Kenya: a household survey. Arch Osteoporos 2020;15:31.

3. Harter DL, Busnello FM, Dibi RP, et al. Association between low bone mass and calcium and caffeine intake among perimenopausal women in Southern Brazil: crosssectional study. Sao Paulo Med J 2013;131:315-22.

4. Du Y, Li P, Wen Y, et al. Evaluating the Correlations Between Osteoporosis and Lifestyle-Related Factors Using Transcriptome-Wide Association Study. Calcif Tissue Int
2020;106:256-63.

5. Kumar A, Kumar V, Rattan V, et al. Secretome proteins regulate comparative osteogenic and adipogenic potential in bone marrow and dental stem cells. Biochimie 2018;155:129-39.

6. Zhu J, Tang H, Zhang Z, et al. Kaempferol slows intervertebral disc degeneration by modifying LPSinduced osteogenesis/adipogenesis imbalance and inflammation response in BMSCs. Int Immunopharmacol 2017;43:236-42.

7. Zhang J, Li Z, Liu W, et al. Effects of bone marrow mesenchymal stem cells transplantation on the recovery of neurological functions and the expression of Nogo-A, $\mathrm{NgR}$, Rhoa, and ROCK in rats with experimentallyinduced convalescent cerebral ischemia. Ann Transl Med 2020;8:390.

8. Li W, Chen R, Lv J, et al. In Vivo Photoacoustic Imaging of Brain Injury and Rehabilitation by High-Efficient Near-Infrared Dye Labeled Mesenchymal Stem Cells with Enhanced Brain Barrier Permeability. Adv Sci (Weinh) 2017;5:1700277.

9. Lin H, Liu T, Li X, et al. The role of gut microbiota metabolite trimethylamine $\mathrm{N}$-oxide in functional impairment of bone marrow mesenchymal stem cells in osteoporosis disease. Ann Transl Med 2020;8:1009.

10. Guo J, Ren R, Yao X, et al. PKM2 suppresses osteogenesis and facilitates adipogenesis by regulating $\beta$-catenin signaling and mitochondrial fusion and fission. Aging (Albany NY) 2020;12:3976-92.

11. Li CJ, Cheng P, Liang MK, et al. MicroRNA-188 regulates age-related switch between osteoblast and adipocyte differentiation. J Clin Invest 2015;125:1509-22.

12. Zhang YH, Li YF, Wang Y, et al. Identification and characterization of N9-methyltransferase involved in converting caffeine into non-stimulatory theacrine in tea. Nat Commun 2020;11:1473.

13. Zhou Y, Guan XX, Zhu ZL, et al. Caffeine inhibits the viability and osteogenic differentiation of rat bone marrowderived mesenchymal stromal cells. Br J Pharmacol 2010;161:1542-52.

14. Xin Z, Wu X, Yu Z, et al. Mechanisms explaining the efficacy of psoralidin in cancer and osteoporosis, a review. Pharmacol Res 2019;147:104334.

15. Pal D, Suman S, Kolluru V, et al. Inhibition of autophagy prevents cadmium-induced prostate carcinogenesis. Br J Cancer 2017;117:56-64.

16. Cao HJ, Li CR, Wang LY, et al. Effect and mechanism of psoralidin on promoting osteogenesis and inhibiting 
Page 14 of 14

adipogenesis. Phytomedicine 2019;61:152860.

17. Zhai Y, Li Y, Wang Y, et al. Psoralidin, a prenylated coumestan, as a novel anti-osteoporosis candidate to enhance bone formation of osteoblasts and decrease bone resorption of osteoclasts. Eur J Pharmacol 2017;801:62-71.

18. Zhai Y, Wang Q, Li Y, et al. The higher osteoprotective activity of psoralidin in vivo than coumestrol is attributed by its presence of an isopentenyl group and through activated PI3K/Akt axis. Biomed Pharmacother 2018;102:1015-24.

19. Li Y, Yang F, Gao M, et al. miR-149-3p Regulates the Switch between Adipogenic and Osteogenic Differentiation of BMSCs by Targeting FTO. Mol Ther Nucleic Acids 2019;17:590-600.

20. Li Y, Feng C, Gao M, et al. MicroRNA-92b-5p modulates melatonin-mediated osteogenic differentiation of bone marrow mesenchymal stem cells by targeting ICAM-1. J Cell Mol Med 2019;23:6140-53.

21. Jiang L, Zhang W, Wei L, et al. Early effects of parathyroid hormone on vascularized bone regeneration and implant osseointegration in aged rats. Biomaterials 2018;179:15-28.

22. Weng H, Huang $H, W u H$, et al. METTL14 Inhibits Hematopoietic Stem/Progenitor Differentiation and Promotes Leukemogenesis via mRNA m6A Modification. Cell Stem Cell 2018;22:191-205.e9.

23. Diederichs $S$, Tuan RS. Functional comparison of humaninduced pluripotent stem cell-derived mesenchymal cells

Cite this article as: Hua R, Zou J, Ma Y, Wang X, Chen Y, Li Y, Du J. Psoralidin prevents caffeine-induced damage and abnormal differentiation of bone marrow mesenchymal stem cells via the classical estrogen receptor pathway. Ann Transl Med 2021;9(15):1245. doi: 10.21037/atm-21-3153
Hua et al. Psoralidin prevents caffeine-induced damage of BMSCs

and bone marrow-derived mesenchymal stromal cells from the same donor. Stem Cells Dev 2014;23:1594-610.

24. Seok JW, Kim D, Yoon BK, et al. Dexras1 plays a pivotal role in maintaining the equilibrium between adipogenesis and osteogenesis. Metabolism 2020;108:154250.

25. Li CJ, Xiao Y, Yang M, et al. Long noncoding RNA Bmncr regulates mesenchymal stem cell fate during skeletal aging. J Clin Invest 2018;128:5251-66.

26. Yang F, Yang L, Li Y, et al. Melatonin protects bone marrow mesenchymal stem cells against iron overloadinduced aberrant differentiation and senescence. J Pineal Res 2017. [Epub ahead of print]. doi: 10.1111/jpi.12422.

27. Kong L, Ma R, Yang X, et al. Psoralidin suppresses osteoclastogenesis in BMMs and attenuates LPS-mediated osteolysis by inhibiting inflammatory cytokines. Int Immunopharmacol 2017;51:31-9.

28. Hao $W$, Zhang $X$, Zhao $W$, et al. Psoralidin induces autophagy through ROS generation which inhibits the proliferation of human lung cancer A549 cells. PeerJ 2014;2:e555.

29. Jin Z, Yan W, Jin H, et al. Psoralidin inhibits proliferation and enhances apoptosis of human esophageal carcinoma cells via NF- $\mathrm{NB}$ and PI3K/Akt signaling pathways. Oncol Lett 2016;12:971-6.

30. Jin Z, Yan W, Jin H, et al. Differential effect of psoralidin in enhancing apoptosis of colon cancer cells via nuclear factor- $\kappa \mathrm{B}$ and B-cell lymphoma-2/B-cell lymphoma2 -associated $\mathrm{X}$ protein signaling pathways. Oncol Lett 2016;11:267-72. 